\title{
Invasive Meningococcal Disease Remains a Health Threat in Vietnam People's Army
}

\author{
Chung Pham Van ${ }^{1,2, *}$ \\ The Trong Nguyen (iD) $2,3, *$ \\ Sy Tien Bui iD 2,4 \\ Trong Van Nguyen ${ }^{2,4}$ \\ Huyen Thi Thanh Tran ${ }^{2,5}$ \\ Dong Trac Pham ${ }^{6}$ \\ Long Phi Trieu ${ }^{7}$ \\ Manh Dang Nguyen ${ }^{1,2}$ \\ 'Department of Foodborne Infectious \\ Disease, Institute of Clinical Infectious \\ Disease, 108 Military Central Hospital, \\ Hanoi, Vietnam; ${ }^{2}$ Vietnamese-German \\ Center of Excellence in Medical \\ Research, 108 Military Central Hospital, \\ Hanoi, Vietnam; ${ }^{3}$ Department of \\ Airborne Infectious Disease and Intensive \\ Care Unit, Institute of Clinical Infectious \\ Disease, 108 Military Central Hospital, \\ Hanoi, Vietnam; ${ }^{4}$ Department of \\ Microbiology, 108 Military Central \\ Hospital, Hanoi, Vietnam; ${ }^{5}$ Department \\ of Molecular Biology, I08 Military Central \\ Hospital, Hanoi, Vietnam; ${ }^{6}$ Military \\ Medical Department, Ministry of National \\ Defense, Hanoi, Vietnam; ${ }^{7}$ Department \\ of Microbiology, Military Institute of \\ Preventive Medicine, Hanoi, Vietnam \\ *These authors contributed equally to \\ this work
}

Objective: Despite strict surveillance, Neisseria meningitidis still causes life-threatening invasive meningococcal disease (IMD). The study aimed to describe the prevalence, clinical and subclinical features, and treatment outcomes of IMD among young soldiers of the Vietnam People's Army.

Methods: A prospective, population-based surveillance study was conducted in all Vietnamese military hospitals from January 2014 to June 2021. The presence of Neisseria meningitidis was confirmed by PCR or culture from blood or/and CSF. Epidemiological indices (incidence, serogroups, and distribution of cases by length of service), medical history, clinical and subclinical features, and treatment outcomes were documented and analyzed.

Results: There were 69 IMD cases (91\% serogroup B) documented, mainly in conscripts (91\%). The highest annual incidence was 3.33/100,000 soldiers per year. Of these cases, 44\% were meningitis $(n=30), 19 \%$ septicemia $(n=13)$, and $38 \%$ meningococcemia $(n=26)$. The most common clinical symptoms were neck stiffness (61 cases, 88\%), petechial rash (51\%), and shock (20 cases, 29\%). Laboratory findings showed leukocytosis in 96\% of IMD cases, PCT $>0.05(\mathrm{ng} / \mathrm{mL})$ in $100 \%$, elevated leukocyte count $(>1,000 / \mathrm{mm} 3)$ in $71 \%$, and high protein $>1 \mathrm{~g} / \mathrm{L}$ in $70 \%$. The overall mortality rate was $9 \%$. Two cases were found to be resistant to ceftriaxone. Prognostic factors of severity included petechial rash $(\mathrm{OR}=9.82, \mathrm{p}<$ $0.001)$, septicemia $(\mathrm{OR}=5.83, \mathrm{p}<0.001)$, meningococcemia $(\mathrm{OR}=6.22, \mathrm{p}<0.001)$, low platelet count, prolonged prothrombin time; high PCT $(\mathrm{AUC}=0.84, \mathrm{p}<0.001)$, and increased creatinine (AUC $=0.86, \mathrm{p}<0.001$ ).

Conclusion: IMD remains a health threat in the armed forces in Vietnam, especially among new recruits. To the best of our knowledge, this is the first study in Vietnam describing ceftriaxone resistance in Neisseria meningitidis and suggests the need to reconsider standard empiric therapy for IMD.

Keywords: meningitis, Neisseria meningitidis, sepsis

\section{Introduction}

Neisseria meningitidis (NM) is one of the most common causes of life-threatening bacterial meningitis worldwide. ${ }^{1}$ These Gram-negative diplococci colonize the nasopharyngeal mucosa of about $10 \%$ of the healthy population. ${ }^{2}$ These pathogens can occasionally cause invasive meningococcal disease (IMD), which can lead to meningitis, septicemia or meningosepticemia with case fatality rates (CFRs) ranging from $4.1 \%$ to $20.0 \%$, depending on age and pathogen strain. ${ }^{3}$ Appropriate and continued monitoring is critical for accurate case management. ${ }^{4}$ After an outbreak of IMD serogroup C between 1977 and 1979, ${ }^{5}$ only a few sporadic cases have been reported, ${ }^{6-8}$ noting the increasing number of NM serogroup $\mathrm{B},{ }^{9}$ while the efficacy of
Correspondence: The Trong Nguyen Department of Airborne Infectious Disease and Intensive Care Unit, Institute of Clinical Infectious Disease, 108 Military Central Hospital, No. I, Tran Hung Dao Street, Hai Ba Trung District, Hanoi, II610, Vietnam

Tel +84964666899

Email drthe108@gmail.com 
current licensed meningococcal $\mathrm{B}$ vaccines in the Asian population remains controversial. ${ }^{10}$

Young children, adolescents, and young adults are the most vulnerable age groups. ${ }^{3,11}$ In Vietnam, high rates of meningococcal infection have been reported among new military recruits, which are associated with a higher risk of developing IMD. Surveys of military units in northern Vietnam revealed high asymptomatic transmission rates of $39 \%$, but only 25 cases of IMD were documented between 2008 and 2014 (5 deaths) (unpublished data). IMD can lead to a fulminant course within a few hours, early treatment, including administration of an appropriate antibiotic, was considered the most effective measure. ${ }^{12}$ Strains with reduced susceptibility to penicillin (PEN) have been reported worldwide, ${ }^{13-16}$ but resistance to third-generation cephalosporins and ciprofloxacin (CIP) was considered rare. ${ }^{15-17}$ Data from Vietnam show a few sporadic cases with NM strains resistant to CIP $(0.19 \mathrm{mg} / \mathrm{L})$ and PEN $(0.25 \mathrm{mg} / \mathrm{L}),{ }^{9}$ whereas resistance to ceftriaxone - a commonly prescribed empiric antibiotic has not been reported.

The aim of this study was to systematically investigate the prevalence, clinical and subclinical features, and treatment outcomes of IMD and to determine prognostic factors for the severity of this devastating infection in young soldiers of the Vietnamese People's Army.

\section{Materials and Methods Designs}

A prospective, military population-based surveillance study was conducted in the Vietnam people's army, by the Department of Military Medicine from January 2014 to June 2021.

\section{Inclusion Criteria}

Case definition (Ministry of Health 2012) was used to include appropriate patients in the study. Enrolled patients were arranged in groups based on where NM was detected. In detail, the detection of NM (either by culture or PCR) only in CSF indicated meningitis; confirmation of NM only in blood (either by culture or PCR) indicated septicemia, and detection of NM in both CSF and blood by PCR or culture indicated meningococcemia. ${ }^{18,19}$

Severe disease was defined as having shock or coma or the need for invasive mechanical ventilation or the need for renal replacement therapy.

\section{Data Collection}

Epidemiological indices (incidence, serogroups, and distribution of cases by service time), medical history, clinical and laboratory findings; treatment (antibiotics, supportive measurements), and outcomes (death, survival, time to defervescence) were collected.

\section{Specimen Collection and Laboratory Testing}

Cerebral spinal fluid (CSF), serum and culture isolates were collected and sent to the Department of Microbiology - Military Institute of Preventive Medicine and 108 Military central Hospital for confirmatory testing and molecular typing.

Gram stain followed by microscopy was used to detect Diplococcic bacteria from clinical samples.

Blood and CSF samples were cultured using BACTEC FX TOP automation systems. The positive bottles were transferred to media agars for conventional culture. In detail, primary cultures were aerobically grown on Blood agar, Chocolate agar and MacConkey agar (Merck, Kenilworth, New Jersey, USA). Once growth of bacteria was detected, bacterial colonies were selected for species' identification using the VITEK MS system and Maldi Tof Vitek MS.

Serogroup was determined by real-time PCR as previously described. ${ }^{20}$

Antibiotic susceptibility was tested using E-test strip (bioMerieux, France) following manufacturer's guidelines. The diameters of the inhibited zones were measured and interpreted following CLSI guidelines. There were no interpretation guidelines for ceftriaxone resistance based on disk diffusion test, therefore, in this study, the diameter of N.meningitidis below $34 \mathrm{~mm}$ was considered an indication of resistance.

\section{Statistical Analysis}

Annual number of active military personnel of 450,000 was used as denominator for calculation of incidence of IMD. ${ }^{21}$ All statistical analyses were computed with R software (version 4.0.5). Where applicable, $95 \%$ confidence intervals are given and a two-sided p-value of $<0.05$ was considered statistically significant. In descriptive analysis: mean \pm standard deviation (SD) was calculated for variables with normal distribution. Chi-squared test was used to compare the frequency of petechial rash, meningitis, septicemia; and MannWhitney test was used where applicable to show the difference between groups of severe and non-severe patients. 
Univariable analysis was used to calculate Odds Ratio (OR). Area Under Curve (AUC) was used to determine the correlation between variables and severity of IMD patients. Variable with AUC $>0.9$ was scaled excellent; from 0.8 to 0.9 was good and from 0.7 to 0.8 was fairly good. ${ }^{22}$

\section{Results \\ Epidemiology}

From 2014 to 2021, there were 69 cases of invasive meningococcal disease (IMD) recorded with mostly meningococcal serotype B (63 cases $91.3 \%)$. The average annual incidence was $1.9 / 100,000$. The incidence was highest with $3.33 / 100,000$ in 2016 . The lowest incidence was $0.22 / 100,000$ in 2021 .

The overall death rate was $8.69 \%(6 / 69)$. The number of deaths was highest in 2016, then declined with no deaths recorded over the last 3 years (2019-2021).

The distribution of IMD cases by army service time and geographical distribution was described in Figures 1 and 2. Nearly half of the cases were new recruits whose service time in military was shorter than 6 months (Figure 1).

There was $65.2 \%(45 / 69)$ of the cases recorded in the Northern Units with the highest number of cases documented in the spring months while in the Southern Units, number of cases was highest in the first months of the rainy season.

\section{Clinical Manifestation and Laboratory Characteristics}

Mean disease onset to hospitalization was $2 \pm 0.9$ days. The most common forms of IMD were meningitis and meningococcemia with $30(43.5 \%)$ and 26 (37.7\%) cases,

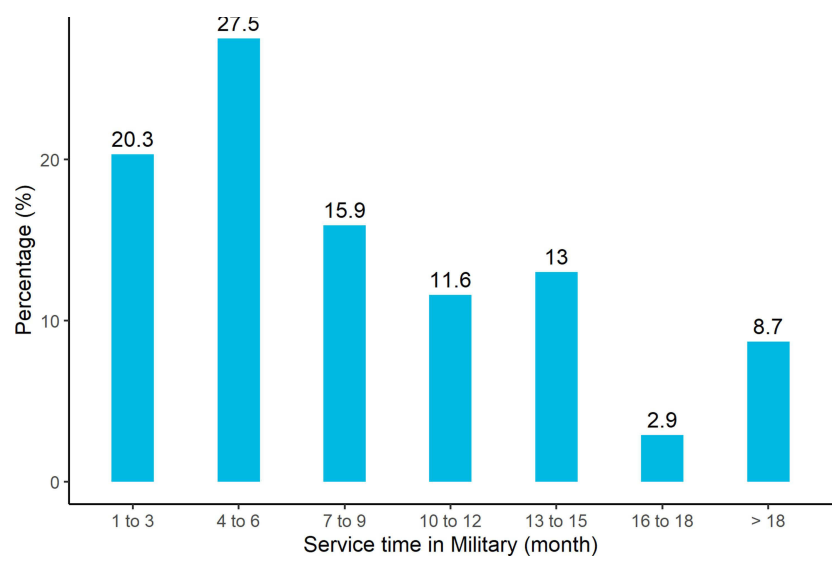

Figure I Distribution of IMD cases by army service time. respectively, while the least common one was septicemia with $13(18.8 \%)$ cases.

Neck stiffness was observed in 61 cases $(88.4 \%)$, most frequently in meningitis group $(100 \%)$ and meningococcemia group (96.2\%), but less commonly found in septicemia group (46.2\%). Altered mental status was documented in $72.5 \%$ of the cases with the highest number in meningococcemia (84\%) followed by septicemia $(69.2 \%)$ and meningitis $(63.3 \%)$. Other neurological symptoms including coma $(18.8 \%)$, seizure $(13 \%)$, and urinary incontinence $(11.6 \%)$ were most commonly documented in septicemia group (30.8\%, 23.1\%, and 15.4, respectively).

Petechial rash was found in $50.7 \%$ of IMD cases with the highest number documented in septicemia group $(92.3 \%)$ compared to that in meningococcemia group (88.5\%). In meningitis group, there were no cases with petechial rash. Shock was described in 20 cases (29\%) in septicemia group.

$95.7 \%$ of IMD cases had WBC $>10 \mathrm{G} / \mathrm{L}, 3$ cases $(23.1 \%)$ in septicemia group had normal or decreased WBC. The majority of IMD cases had PCT level $>10$ (ng/mL) with $62.5 \%, 90.9 \%$, and $66.7 \%$ in meningitis, septicemia and meningococcemia group, respectively.

Thrombocytopenia (platelets $<100 \mathrm{G} / \mathrm{L}$ ) was documented in $37.7 \%$ of IMD cases, with the highest number documented in septicemia group (69.2\%). There was no case with platelets $<100 \mathrm{G} / \mathrm{L}$ in meningitis group. Prolonged prothrombin time was found in two thirds of IMD cases with the highest number documented in meningococcemia group (81.8\%).

Elevated serum bilirubin ( $>17 \mu \mathrm{mol} / \mathrm{L})$ was documented in $50 \%, 34.8 \%$, and $48 \%$ of IMD cases in septicemia, meningitis and meningococcemia group, respectively. Renal failure (serum creatinine $>120 \mu \mathrm{mol} / \mathrm{L}$ ) was found in one third of IMD cases with the highest number documented in septicemia group $(76.9 \%)$ followed by meningococcemia group (26.9\%) and only 1 case $(3.3 \%)$ in meningitis group.

Elevated WBC count $(>1,000 / \mathrm{mm} 3)$ was found in $70.6 \%$ of IMD case; high protein level $(>1 \mathrm{~g} / \mathrm{L})$ was documented in $70 \%$ of cases; low CSF glucose level (glucose $\mathrm{CSF} /$ glucose serum $<0.6$ ) was found in $80 \%$ of cases.

\section{Treatment and Outcomes}

About one third of patients were treated with intravenous (IV) 3rd generation cephalosporin before admission and carbapenem was empirically given to 3 cases (4.3\%).

Antibiotic susceptibility tests were done for 8 isolates and revealed 2 cases with resistance to ceftriaxone (first line to treat IMD). 


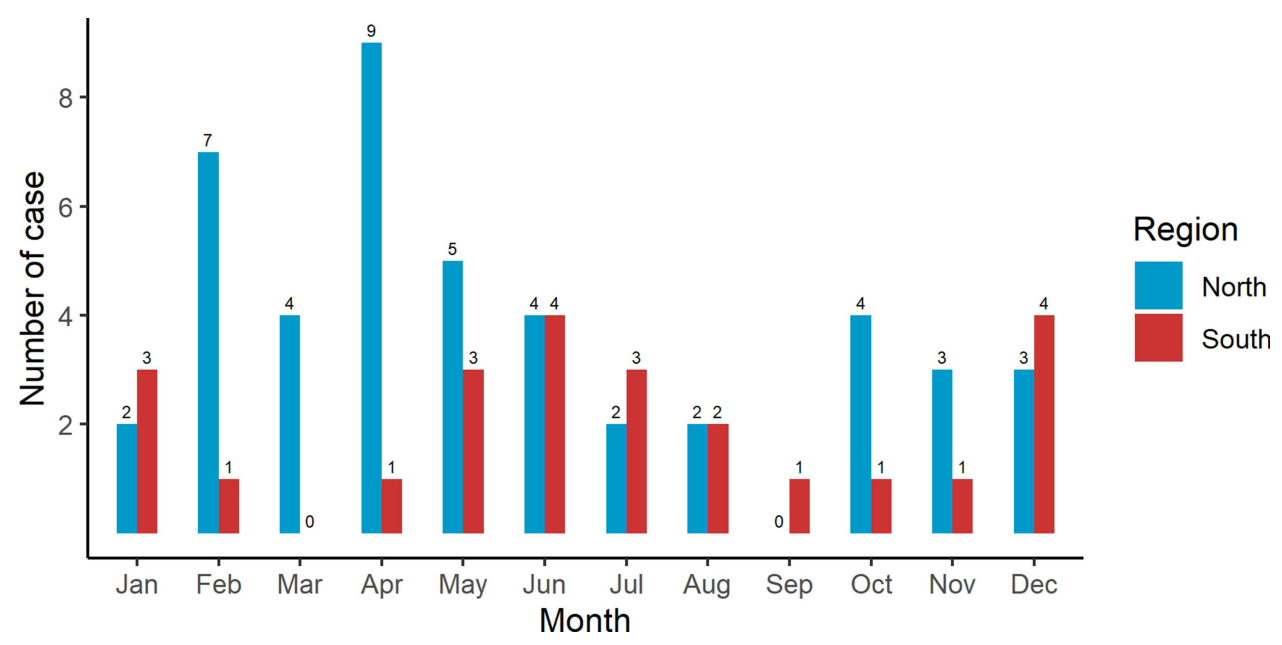

Figure 2 Number of IMD cases.

One third of the patients needed central catheter insertion with the highest number documented in meningococcemia group (50\%). $13 \%$ of cases needed mechanical ventilation. Renal replacement therapy was done for $8.7 \%$ of cases.

Overall, there were 6 deaths $(8.69 \%)$ due to IMD.

Petechial rash $(\mathrm{OR}=9.82)$, septicemia $(\mathrm{OR}=5.83)$, meningococcemia $(\mathrm{OR}=6.22)$, low platelet count, prolonged prothrombin time, high PCT level (AUC = 0.84 ), increased creatinine (AUC $=0.86$ ) were shown to be significant prognostic factors for severe IMD (Table 1).

\section{Discussion}

This study, the first of its kind in Vietnam, provides comprehensive insight into the prevalence and burden of invasive meningococcal disease in the military. Overall, the average annual incidence of IMD was $1.9 / 100,000$ (Table 2). This is higher compared to what was reported in European countries $(0.7 / 100,000)$, the United States $(0.12 / 100,000)$, and Canada $(0.3 / 100,000)$ and some other countries: China, Taiwan, Japan, South Korea. ${ }^{11}$

The highest IMD incidence was documented in 2016 with 3.33 cases per 100,000 soldiers and 2 deaths (Table 2). In response to this rising threat of IMD, the

Table I Common Prognostic Factors in IMD

\begin{tabular}{|c|c|c|c|c|}
\hline Variables & $\begin{array}{l}\text { Non-Severe } \\
(\mathrm{N}=42)\end{array}$ & $\begin{array}{l}\text { Severe } \\
(N=27)\end{array}$ & $\mathbf{p}$ & \\
\hline Petechial rash & 13 (31\%) & 22 (8I.5\%) & $<0.001 *$ & $O R=9.82$ \\
\hline Meningitis & $26(61.9 \%)$ & $4(14.8 \%)$ & \multirow[t]{3}{*}{$<0.001 *$} & $O R=1$ \\
\hline Septicemia & $3(7.1 \%)$ & $10(37 \%)$ & & $\mathrm{OR}=5.83$ \\
\hline Meningococcemia & 13 (31\%) & $13(48.1 \%)$ & & $O R=6.22$ \\
\hline WBC (G/L) & $20.9[10.4-38.6]$ & $21.3[1.36-50.1]$ & $0.7962^{\#}$ & \\
\hline Bilirubin $(\mu \mathrm{mol} / \mathrm{L})$ & $13.9[4.1-44.6]$ & $19.6[2.9-47.4]$ & $0.1174^{\#}$ & \\
\hline Platelets (G/L) & 144 [14-305] & $37[|8-| 7 \mid]$ & $<0.001^{\#}$ & $A \cup C=0.15$ \\
\hline Prothrombin (\%) & $62[8-96]$ & $25[\mathrm{II}-38]$ & $<0.001^{\#}$ & $\mathrm{AUC}=0.20$ \\
\hline Creatinine $(\mu \mathrm{mol} / \mathrm{L})$ & $83[60-199]$ & $\mid 70$ [56-49|] & $<0.001^{\#}$ & $A \cup C=0.86$ \\
\hline PCT (ng/mL) & $24.465[0.08-102]$ & $100[100-200]$ & $<0.001^{\#}$ & $A \cup C=0.84$ \\
\hline
\end{tabular}

Notes: *Chi-squared test; "Mann-Whitney test.

Abbreviations: WBC, white blood count; PCT, procalcitonin. 
Table 2 Incidence, Serogroup, and Mortality

\begin{tabular}{|l|l|l|l|l|l|l|}
\hline \multirow{2}{*}{ Year } & \multicolumn{2}{|l|}{ Case } & Incidence* & \multicolumn{2}{l|}{ Serogroup } & Mortality \\
\cline { 2 - 7 } & Number & & B & W I35 & NA \\
\hline 2014 & & 2 & 08 & 01 & & \\
\hline 2015 & 9 & 1.78 & 08 & & 01 & 2 \\
\hline 2016 & 8 & 3.33 & 13 & 01 & 02 & 2 \\
\hline 2017 & 15 & 2.67 & 12 & 01 & 1 \\
\hline 2018 & 12 & 2.67 & 11 & & 1 \\
\hline 2019 & 12 & 2 & 09 & & \\
\hline 2020 & 9 & 0.67 & 03 & 1 & & \\
\hline 2021 & 1 & 0.22 & 1.92 & $63(91.30 \%)$ & $02(2.90 \%)$ & $04(5.80 \%)$ \\
\hline Sum (\%) & $69(100 \%)$ & & & $6(8.69 \%)$ \\
\hline
\end{tabular}

Notes: *Incidence (cases//00,000 soldiers/year); NA (serogroup was not identified).

Department of Military Medicine released a new guideline for management of suspected cases of IMD. Thereafter, the number of cases decreased gradually and the lowest number of cases -0.22 per 100,000 soldiers - was documented in 2021, with only 1 death in the four most recent years (Table 2). The lowest incidence of IMD recorded in the last 2 years of the study time overlapped with the occurrence of the Covid-19 pandemic when people, especially military men, are being asked to wear facemasks and strictly follow sanitation guidelines. These control measures were believed to limit the spread of airborne pathogens including Neisseria meningitidis. ${ }^{23}$ Our data suggest that, in low-income settings with limited access to vaccines, using facemasks and practicing hand hygiene regularly could be practical and efficient to prevent invasive meningococcal disease.

The number of cases was high in the first 3-months group (Figure 1), when newly recruited soldiers gathered in military units, lived in crowded conditions, ${ }^{24,25}$ and were exposed to Neisseria meningitis. The number of IMD cases was lower in other groups of subjects: 16-18 months of service (2.9\%) and more than 18 months of service (8.7\%) (Figure 1) whose protective immunity against Neisseria meningitis was demonstrated to be more sufficient. ${ }^{26}$ This annual trend of prevalence and incidence is similar to what was reported elsewhere ${ }^{24}$ and suggests the prevention strategy should focus on the youngest cohort as the first priority.

Mild IMD manifestations can progress to severe symptoms rapidly with mean duration from the first symptom onset to hospitalization of $2 \pm 0.9$ days, and even shorter in septicemia group (1.8 \pm 0.8 days) (Table 3$)$. Similar rapid progression was documented in a cohort of 752 patients in the Netherlands. ${ }^{27}$ Therefore, early diagnosis of IMD and hourly monitoring of severe signs and symptoms, even if the patient initially looks well at the onset of IMD, are crucial for optimal treatment and to minimize number of deaths.

Shock was documented in $29 \%(20 / 69)$ of the cases (Table 3), higher than that reported in another study, ${ }^{27}$ but lower than data from a cohort study of IMD in young adults in the US during the 1990s (117/278 cases). ${ }^{28}$ Shock in meningococcal sepsis is caused by hypovolemia (result of capillary leak syndrome), ${ }^{29}$ myocardial dysfunction, ${ }^{30}$ altered vasomotor tone and adrenal insufficiency in some cases. ${ }^{31}$ Shock was one of the factors that defined severe disease (Table 1) and was associated with higher mortality. ${ }^{18,27}$

Classical symptoms remain highly common in IMD despite the low specificity for bacterial meningitis. ${ }^{32}$ Neck stiffness is the most common symptom (61 cases, $88.4 \%$ ) appearing in $100 \%$ of cases in meningitis group and noticeably in $46.2 \%$ of cases in septicemia group (Table 3).

Altered mental status $(72.5 \%)$ and other neurological symptoms including seizure (13\%), and urinary incontinence (11.6\%) are less common (Table 3). Laboratory findings including elevated WBC count and PCT level (Table 4) are valuable parameters that, together with classical symptoms, help establish the diagnosis of IMD. Petechial skin rash is frequently observed in $\mathrm{IMD}^{32,33}$ and could possibly lead to 
Table 3 Clinical Manifestation of IMD

\begin{tabular}{|l|l|l|l|l|}
\hline & Meningitis N = 30 (43.5\%) & Septicemia N = I3 (18.8\%) & Meningococcemia N = 26 (37.7\%) & Sum 69 (100\%) \\
\hline Admission & $1.9 \pm 0.9$ & $1.8 \pm 0.8$ & $2.2 \pm 0.8$ & $2 \pm 0.9$ \\
\hline AMS & $19(63.3 \%)$ & $9(69.2 \%)$ & $22(84 \%)$ & $50(72.5 \%)$ \\
\hline Coma & $4(13.3 \%)$ & $4(30.8 \%)$ & $5(19.2 \%)$ & $13(18.8 \%)$ \\
\hline Neck stiffness & $30(100 \%)$ & $6(46.2 \%)$ & $25(96.2 \%)$ & $61(88.4 \%)$ \\
\hline Seizure & $4(13.3 \%)$ & $3(23.1 \%)$ & $2(7.7 \%)$ & $9(13 \%)$ \\
\hline Incontinence & $4(13.3 \%)$ & $2(15.4 \%)$ & $2(7.7 \%)$ & $8(11.6 \%)$ \\
\hline Shock & 0 & $10(76.9 \%)$ & $10(38.5 \%)$ & $20(29 \%)$ \\
\hline Petechial rash & 0 & $12(92.3 \%)$ & $23(88.5 \%)$ & $36(50.7 \%)$ \\
\hline
\end{tabular}

Notes: Admission: time from onset to hospitalization (days); incontinence: urinary incontinence.

Abbreviation: AMS, altered mental status.

Table 4 Laboratory Findings

\begin{tabular}{|c|c|c|c|c|c|}
\hline \multicolumn{2}{|l|}{ Variables } & Meningitis $\mathbf{N}=\mathbf{3 0}$ & Septicemia $N=13$ & Meningococcemia $N=26$ & Sum $\mathbf{N}=69$ \\
\hline \multicolumn{6}{|l|}{ Blood analysis } \\
\hline \multirow{4}{*}{$\begin{array}{l}\text { WBC (G/L) } \\
(N=65)\end{array}$} & $<4$ & 0 & I $(7.7 \%)$ & 0 & $\mathrm{I}(\mathrm{l} .4 \%)$ \\
\hline & $4-10$ & 0 & $2(15.4 \%)$ & 0 & $2(2.9 \%)$ \\
\hline & $>10$ & $30(100 \%)$ & 10 (76.9\%) & $26(100 \%)$ & $66(95.7 \%)$ \\
\hline & Mean & $22.3 \pm 6.3$ & $18.9 \pm 11.5$ & $24.2 \pm 9$ & $22.4 \pm 8.6$ \\
\hline \multirow{4}{*}{$\begin{array}{l}\text { PCT }(\mathrm{ng} / \mathrm{mL}) \\
(\mathrm{N}=34)\end{array}$} & $\leq 0.05$ & 0 & 0 & 0 & 0 \\
\hline & $>0.05-2$ & $5(20.8 \%)$ & I (9.1\%) & $2(8.3 \%)$ & $8(13.6 \%)$ \\
\hline & $>2-10$ & $4(16.7 \%)$ & 0 & $6(25 \%)$ & $10(16.9 \%)$ \\
\hline & $>10$ & 15 (62.5\%) & 10 (90.9\%) & $16(66.7 \%)$ & 41 (69.5\%) \\
\hline \multicolumn{2}{|c|}{ PLT count $<100 \mathrm{G} / \mathrm{L}$} & 0 & $9(69.2 \%)$ & 17 (65.4\%) & $26(37.7 \%)$ \\
\hline \multicolumn{2}{|c|}{ Prothrombin $<70 \%$} & $10(47.6 \%)$ & $8(80 \%)$ & $18(81.8 \%)$ & $36(67.9 \%)$ \\
\hline \multicolumn{2}{|c|}{ Bilirubin $>17 \mu \mathrm{mol} / \mathrm{L}$} & $8(34.8 \%)$ & $5(50 \%)$ & $12(48 \%)$ & $25(43.1 \%)$ \\
\hline \multicolumn{2}{|c|}{ Creatinine $>120 \mu \mathrm{mol} / \mathrm{L}$} & I (3.3\%) & $10(76.9 \%)$ & $7(26.9 \%)$ & $18(26.1 \%)$ \\
\hline \multicolumn{6}{|l|}{ CSF analysis } \\
\hline \multirow[t]{3}{*}{ WBC (G/L) } & $<500$ & I (3.3\%) & $6(100 \%)$ & $5(19.2 \%)$ & $12(19.4 \%)$ \\
\hline & $500-1000$ & $3(10 \%)$ & 0 & $2(7.7 \%)$ & $5(8.1 \%)$ \\
\hline & $>1000$ & 26 (88.7\%) & 0 & 19 (73.1\%) & 45 (72.6\%) \\
\hline \multicolumn{2}{|l|}{ Neutrophilia } & 26 (89.7\%) & & 22 (84.6\%) & 48 (87.3\%) \\
\hline \multirow[t]{3}{*}{ Protein $(g / L)$} & $<1$ & $5(16.7 \%)$ & $6(100 \%)$ & 7 (29.2\%) & $18(30 \%)$ \\
\hline & $1-3$ & 12 (40\%) & 0 & 9 (37.5\%) & 21 (35\%) \\
\hline & $>3$ & $13(43.3 \%)$ & 0 & 8 (33.3\%) & $21(35 \%)$ \\
\hline \multicolumn{2}{|c|}{ Glucose ratio $<0.6$} & $27(90 \%)$ & 0 & $21(87 \%)$ & $48(80 \%)$ \\
\hline
\end{tabular}

Notes: Neutrophilia: neutrophil percentage $>70 \%$, glucose ratio (glucose in CSF/glucose in serum).

Abbreviations: WBC, white blood cell count; PCT, procalcitonin; PLT, platelet; CSF, cerebrospinal fluid. 
Table 5 Treatment and Outcomes

\begin{tabular}{|c|c|c|c|c|}
\hline Variables & $\begin{array}{l}\text { Meningitis } \\
N=30 \text { (43.5\%) }\end{array}$ & $\begin{array}{l}\text { Septicemia } \\
N=13(18.8 \%)\end{array}$ & $\begin{array}{l}\text { Meningococcemia } \\
N=26(37.7 \%)\end{array}$ & $\begin{array}{l}\text { Sum } \\
69(100 \%)\end{array}$ \\
\hline $3 G C$ & $9(30 \%)$ & $3(23.1 \%)$ & 9 (34.6\%) & 21 (30.4\%) \\
\hline Carbapenem & 0 & 2 (I5.4\%) & I (3.8\%) & $3(4.3 \%)$ \\
\hline Central line & 4 (I3.3\%) & 6 (46.2\%) & $13(50 \%)$ & $23(33.3 \%)$ \\
\hline Arterial line & 0 & 0 & I (3.8\%) & I (I.4\%) \\
\hline CRRT & 0 & $2(15.4 \%)$ & $4(15.4 \%)$ & $6(8.7 \%)$ \\
\hline MV & I (3.3\%) & $2(15.4 \%)$ & $6(23.1 \%)$ & $9(13 \%)$ \\
\hline FFP transfusion & I (3.3\%) & 2 (15.3\%) & I (3.8\%) & $4(5.8 \%)$ \\
\hline PLT transfusion & 0 & I (7.7\%) & I (3.8\%) & $2(2.9 \%)$ \\
\hline Amputation & 0 & 2 (15.4\%) & 0 & 2 (2.9\%) \\
\hline Mortality & 0 & $4(30.8 \%)$ & 3 (1 1.5\%) & $6(8.7 \%)$ \\
\hline Defervescence & $4 \pm 1.9$ & $6 \pm 4.5$ & $4.9 \pm 2.8$ & $4.7 \pm 2.8$ \\
\hline
\end{tabular}

Abbreviations: 3GC, 3rd Generation Cephalosporin use before admission; CRRT, continuous renal replacement therapy; MV, mechanical ventilation; FFP, fresh frozen plasma transfusion; PLT transfusion, platelet transfusion.

misdiagnosis, for instance, with Dengue hemorrhagic fever in this cohort. This could consequently lead to delayed antibiotic treatment and increased mortality. The primary pathophysiology causing meningococcal petechial rash is disorders of coagulation. ${ }^{29,31,34}$ Thrombocytopenia (platelets $<100 \mathrm{G} / \mathrm{L}$ ) and prolonged prothrombin time are common disorders (Table 4) and are associated with severe disease (Table 1). ${ }^{35,36}$

Renal failure is most frequently found in septicemia cases and related to the severity of shock ${ }^{29}$ which may require continuous renal replacement therapy (CRRT) (Table 5).

The preferred empirical treatment of IMD includes ceftriaxone. ${ }^{18}$ However, the low availability of this drug in infirmary of all military units in Vietnam ${ }^{6}$ may be related to its limited use before hospitalization (Table 5). In addition, due to an increasing prevalence of antimicrobial resistance in Vietnam, carbapenem is empirically recommended for treatment of severe cases at admission to central hospitals, ${ }^{37}$ as exemplified by 3 cases in our cohort (Table 5).

Susceptibility assay revealed two culture isolates with ceftriaxone resistant NM. These two cases were successfully treated with a combination of ceftriaxone and IV ciprofloxacin (ceftriaxone use was prolonged upon decision of clinician). This is of great concern because to the best of our knowledge, isolates with reduced susceptibility to third generation cephalosporin are rare. ${ }^{14-17}$ Another study in civil patients in Southern Vietnam revealed 3 out of 11 isolates were resistant and 5 out of 8 isolates intermediated to CIP $(0.19 \mathrm{mg} / \mathrm{L})$ and PEN $(0.25 \mathrm{mg} / \mathrm{L})$, respectively. ${ }^{9}$ We assumed the efficacy of the treatment was maintained by ciprofloxacin.

In conclusion, IMD remains a health threat in the military in Vietnam especially in new military recruits. To the best of our knowledge, this is the first study to describe ceftriaxone-resistant Neisseria meningitidis in Vietnam, however, further studies are needed to investigate this phenomenon.

\section{Ethics Approval}

The study was approved by the Ethical Committee of 108 Military Central Hospital (No 432/ HĐĐĐ). The consent obtained from conscious patients or legal guardians of unconscious patients was written informed consent. This study was conducted in accordance with the Declaration of Helsinki.

\section{Acknowledgments}

We are thankful to all patients who voluntarily participated in this study, staff of the Department of Military Medicine for their generous support of this study. Chung Pham Van and The Trong Nguyen are co-first authors for this study.

\section{Author Contributions}

All authors contributed to data analysis, drafting or revising the article, have agreed on the journal to which the 
article will be submitted, gave final approval of the version to be published, and agree to be accountable for all aspects of the work.

\section{Disclosure}

The authors report no conflicts of interest in this work.

\section{References}

1. Oordt-Speets AM, Bolijn R, van Hoorn RC, Bhavsar A, Kyaw MH. Global etiology of bacterial meningitis: a systematic review and meta-analysis. PLoS One. 2018;13(6):e0198772. doi:10.1371/journal.pone. 0198772

2. Serra L, Presa J, Christensen H, Trotter C. Carriage of Neisseria Meningitidis in low and middle income countries of the Americas and Asia: a review of the literature. Infect Dis Ther. 2020;9 (2):209-240. doi:10.1007/s40121-020-00291-9

3. Wang B, Santoreneos R, Giles L, Afzali HHA, Marshall H. Case fatality rates of invasive meningococcal disease by serogroup and age: a systematic review and meta-analysis. Vaccine. 2019;37 (21):2768-2782. doi:10.1016/j.vaccine.2019.04.020

4. Li J, Shao Z, Liu G, et al. Meningococcal disease and control in China: findings and updates from the Global Meningococcal Initiative (GMI). $J$ Infect. 2018;76(5):429-437.

5. Oberti J, Hoi N, Caravano R, Tan C, Roux J. An epidemic of meningococcal infection in Vietnam (southern provinces). Bull World Health Organ. 1981;59(4):585-590.

6. Viet NT, Nam NM, Trung NV, Van Tong H. Meningococcal disease in one soldier patient: case report from Vietnam. Vietnam $J \mathrm{Sci}$ Technol Eng. 2021;63(1):54-58. doi:10.31276/VJSTE.63(1).54-58

7. Le TT, Tran TX, Trieu LP, Austin CM, Nguyen HM, Van Quyen D. Genotypic characterization and genome comparison reveal insights into potential vaccine coverage and genealogy of Neisseria meningitidis in military camps in Vietnam. PeerJ. 2020;8:e9502. doi:10.7717/peerj.9502

8. Taylor WR, Nguyen K, Nguyen D, et al. The spectrum of central nervous system infections in an adult referral hospital in Hanoi, Vietnam. PLoS One. 2012;7(8):e42099. doi:10.1371/journal. pone. 0042099

9. Phan T, Ho N, Vo D, et al. Characterization of Neisseria meningitidis in Vietnam from 1980s-2019. Int J Infect Dis. 2020;101:147. doi:10.1016/j.ijid.2020.09.399

10. Chen M, Rodrigues CM, Harrison OB, et al. Invasive meningococcal disease in Shanghai, China from 1950 to 2016: implications for serogroup B vaccine implementation. Sci Rep. 2018;8(1):1-9. doi:10.1038/s41598-017-17765-5

11. Purmohamad A, Abasi E, Azimi T, et al. Global estimate of Neisseria meningitidis serogroups proportion in invasive meningococcal disease: a systematic review and meta-analysis. Microb Pathog. 2019;134:103571. doi:10.1016/j.micpath.2019.103571

12. Stephens DS, Greenwood B, Brandtzaeg P. Epidemic meningitis, meningococcaemia, and Neisseria meningitidis. Lancet. 2007;369 (9580):2196-2210. doi:10.1016/S0140-6736(07)61016-2

13. Antignac A, Boneca IG, Rousselle J-C, et al. Correlation between alterations of the penicillin-binding protein 2 and modifications of the peptidoglycan structure in Neisseria meningitidis with reduced susceptibility to penicillin G. J Biol Chem. 2003;278(34):31529-31535. doi:10.1074/jbc.M304607200

14. Bennett DE, Meyler K, Cafferkey M, Cunney R. Antibiotic susceptibility and molecular analysis of invasive Neisseria meningitidis recovered in the Republic of Ireland, 1996 to 2016. Eur J Clin Microbiol Infect Dis. 2021;40(6):1127-1136. doi:10.1007/s10096020-04114-0
15. Gorla MC, Pinhata JMW, Dias UJ, de Moraes C, Lemos AP. Surveillance of antimicrobial resistance in Neisseria meningitidis strains isolated from invasive cases in Brazil from 2009 to 2016. J Med Microbiol. 2018;67(6):750-756. doi:10.1099/jmm.0.000743

16. Zouheir Y, Atany T, Boudebouch N. Emergence and spread of resistant N. meningitidis implicated in invasive meningococcal diseases during the past decade (2008-2017). $J$ Antibiot. 2019;72(3):185-188. doi:10.1038/s41429-018-0125-0

17. Deghmane A-E, Hong E, Taha M-K. Emergence of meningococci with reduced susceptibility to third-generation cephalosporins. J Antimicrob Chemother. 2016;72(1):95-98. doi:10.1093/jac/dkw400

18. McGill F, Heyderman R, Michael B, et al. The UK joint specialist societies guideline on the diagnosis and management of acute meningitis and meningococcal sepsis in immunocompetent adults. $J$ Infect. 2016;72(4):405-438.

19. Clark SA, Campbell H, Lucidarme J, Borrow R, Ladhani SN. UK guidelines and testing for invasive meningococcal disease. Lancet Infect Dis. 2021;21(4):455-456. doi:10.1016/S1473-3099(21)00092$\mathrm{X}$

20. WHO, CDC. Laboratory Methods for the Diagnosis of Meningitis. 2nd ed.; 2011:323.

21. Thayer CA. Vietnam People's Army: Development and Modernization. Citeseer; 2009.

22. DeLong ER, DeLong DM, Clarke-Pearson DL. Comparing the areas under two or more correlated receiver operating characteristic curves: a nonparametric approach. Biometrics. 1988;44(3):837-845. doi: $10.2307 / 2531595$

23. Brueggemann $\mathrm{AB}$, van Rensburg MJJ, Shaw D, et al. Changes in the incidence of invasive disease due to Streptococcus pneumoniae, Haemophilus influenzae, and Neisseria meningitidis during the COVID-19 pandemic in 26 countries and territories in the Invasive Respiratory Infection Surveillance Initiative: a prospective analysis of surveillance data. Lancet Digit Health. 2021;3(6):e360-e370. doi:10.1016/S2589-7500(21)00077-7

24. Christopher GW. Invasive meningococcal disease at Fort Ord and San Diego Naval Training Center, 1962-1964. Mil Med. 2020;185(78):397-399. doi:10.1093/milmed/usz389

25. Archer BN, Chiu CK, Jayasinghe SH, et al. Epidemiology of invasive meningococcal B disease in Australia, 1999-2015: priority populations for vaccination. Med $J$ Aust. 2017;207(9):382-387. doi: $10.5694 / \mathrm{mja} 16.01340$

26. Goldschneider I, Gotschlich EC, Artenstein MS. Human immunity to the meningococcus: i. The role of humoral antibodies. J Exp Med. 1969;129(6):1307-1326. doi:10.1084/jem.129.6.1307

27. De Greeff S, De Melker H, Schouls L, Spanjaard L, Van Deuren M. Pre-admission clinical course of meningococcal disease and opportunities for the earlier start of appropriate intervention: a prospective epidemiological study on 752 patients in the Netherlands, 20032005. Eur J Clin Microbiol Infect Dis. 2008;27(10):985-992. doi:10.1007/s10096-008-0535-1

28. Harrison LH, Pass MA, Mendelsohn AB, et al. Invasive meningococcal disease in adolescents and young adults. JAMA. 2001;286 (6):694-699. doi:10.1001/jama.286.6.694

29. Pathan N, Faust S, Levin M. Pathophysiology of meningococcal meningitis and septicaemia. Arch Dis Child. 2003;88(7):601-607. doi:10.1136/adc.88.7.601

30. Boucek MM, Boerth RC, Artman M, Graham TP Jr, Boucek RJ Jr. Myocardial dysfunction in children with acute meningococcemia. $J$ Pediatr. 1984;105(4):538-542. doi:10.1016/S0022-3476(84) 80416-3

31. Wu M-Y, Chen C-S, Tsay C-Y, Yiang G-T, Ke J-Y, Lin P-C. Neisseria meningitidis induced fatal Waterhouse-Friderichsen syndrome in a patient presenting with disseminated intravascular coagulation and multiple organ failure. Brain Sci. 2020;10(3):171. doi:10.3390/brainsci10030171 
32. Granier S, Owen P, Pill R, Jacobson L. Recognising meningococcal disease in primary care: qualitative study of how general practitioners process clinical and contextual information. BMJ. 1998;316 (7127):276-279. doi:10.1136/bmj.316.7127.276

33. Hazarika RD, Deka NM, Khyriem A, et al. Invasive meningococcal infection: analysis of 110 cases from a tertiary care centre in North East India. Indian J Pediatr. 2013;80(5):359-364. doi:10.1007/ s12098-012-0855-0

34. McGrath A, Barrett MJ. Petechiae. StatPearls Publishing; 2018.

35. Martínez DM, Aguilera-Alonso D, Mancebo JG, et al. Invasive meningococcal disease in children and adults in a tertiary level hospital. Recent epidemiology and prognostic factors. An Pediatr. 2019;91(5):296-306. doi:10.1016/j.anpede.2019.09.001
36. Amarilla S, Lovera D, Martínez-de Cuellar C, et al. Epidemiology, clinical and prognostic factors of invasive meningococcal disease in a reference center in Paraguay. 2005 to 2018. Rev Inst Med Trop. 2018;13(1):40-53. doi:10.18004/imt/201813140-53

37. Rhodes A, Evans LE, Alhazzani W, et al. Surviving sepsis campaign: international guidelines for management of sepsis and septic shock: 2016. Intensive Care Med. 2017;43(3):304-377. doi:10.1007/s00134017-4683-6

\section{Publish your work in this journal}

Infection and Drug Resistance is an international, peer-reviewed openaccess journal that focuses on the optimal treatment of infection (bacterial, fungal and viral) and the development and institution of preventive strategies to minimize the development and spread of resistance. The journal is specifically concerned with the epidemiology of antibiotic resistance and the mechanisms of resistance development and diffusion in both hospitals and the community. The manuscript management system is completely online and includes a very quick and fair peerreview system, which is all easy to use. Visit http://www.dovepress.com/ testimonials.php to read real quotes from published authors. 